\title{
Biological and Genetic Characterization of New and Known Necroviruses Causing an Emerging Systemic Necrosis Disease of Corn Salad (Valerianella locusta) in France
}

\author{
E. Verdin, A. Marais, C. Wipf-Scheibel, C. Faure, B. Pelletier, P. David, L. Svanella-Dumas, \\ C. Poisblaud, H. Lecoq, and T. Candresse ${ }^{\dagger}$
}

First, third, and ninth authors: Unité de Pathologie Végétale, INRA, Domaine Saint Maurice, 67, allée des chênes, CS 60094, F84143 Montfavet Cedex, France; second, fourth, seventh, and tenth authors: Equipe de Virologie, UMR 1332 BFP, INRA, University of Bordeaux, 71 Avenue Edouard Bourleaux, CS20032, F33882 Villenave d'Ornon Cedex, France; fifth author: Comité Départemental de Développement Maraîcher (CDDM), Maisons des maraîchers, La métairie neuve, F44860 Pont St Martin, France; and sixth and eighth authors: HM.CLAUSE, 1 Chemin du Moulin des Ronzières, 49800 La Bohalle, France.

Accepted for publication 21 February 2018.

\begin{abstract}
An emerging systemic necrosis disease of corn salad was first observed in the Nantes region of France in the late 2000s. Classical virology and highthroughput sequencing approaches demonstrated that the disease is associated with four different necroviruses: tobacco necrosis virus A (TNVA), tobacco necrosis virus D (TNVD), olive mild mosaic virus (OMMV), and a novel recombinant Alphanecrovirus for which the name corn salad necrosis virus (CSNV) is proposed. Satellite tobacco necrosis virus was also frequently observed. Koch's postulates were completed for all four agents,

corn salad. OMMV was the most frequently observed virus and causes the most severe symptoms. TNVA was the second, both in terms of prevalence and symptom severity while TNVD and CSNV were only rarely observed and caused the less severe symptoms. The emergence of this systemic disease may have been favored by the short and repeated cropping cycles used for corn salad, possibly allowing the selection of necrovirus isolates with an improved ability to systemically invade this specialty crop.
\end{abstract} each one alone being able to cause systemic necrosis of varying severity in
Additional keywords: etiology, next generation sequencing.
Corn salad (Valerianella locusta L.), otherwise known as lamb's lettuce, mâche, Doucette, or rapunzel, is a small annual plant of the family Caprifolicaeae. Its dark green leaves have a characteristic nutty flavor and are popularly eaten as specialty salad greens in several countries. It grows wild in parts of Europe, northern Africa, and western Asia and is a common weed in cultivated lands. It was originally foraged by Europeans before being introduced in cultivation (Peron and Rees 1998; Wikipedia 2017). As a crop, it is intensively grown in a small region around the city of Nantes in the West of France, which is the primary producer of corn salad in Europe. France is the leading producer in the world with about 43,000 tons produced on a little under 8,000 ha in 2014 , and about $80 \%$ of the French production (equivalent to about $50 \%$ of the European production) comes from the Nantes region alone (Peron and Rees 1998).

Corn salad is today a high value crop produced mostly by highly specialized growers. Being a hardy plant, it is grown year around, in

${ }^{\dagger}$ Corresponding author: T. Candresse; E-mail: thierry.candresse@inra.fr

Funding: The financial contribution of the Conseil Régional des Pays de Loire, of France Agrimer and of the Ministry of Agriculture through the CASDAR program MASAMA to CCDM is gratefully acknowledged.

First and second authors contributed equally to this work.

The nucleotide sequences reported here have been deposited in GenBank under accession numbers KX906929, KX906928, KX906930, and MF125267 for complete genome sequences and KX944577 to KX944610 plus KX944613 to KX944628 for the partial sequences used in diversity analyses.

*The $\boldsymbol{e}$-Xtra logo stands for "electronic extra" and indicates that three supplementary tables are published online.

(C) 2018 The American Phytopathological Society open fields or under plastic houses, with a production peak from September to April. The cropping cycle is short as very young tender plants are harvested when they reach the four to six leaf stage (Peron and Rees 1998). Several crops are produced on the same plot per year, with either successive crops of corn salad alone or alternating with other specialty short cycle crops such as radish, young lettuce, young beet greens or rucolla. Harvesting, washing, and conditioning for fresh, ready to use salad bags are highly mechanized, necessitating a perfect visual and sanitary status of harvested plants in order to obtain irreproachable production lots.

Starting in 2008 in the Nantes region, a new disease was observed. Symptoms developed during the last period before harvest, when plants reach the four to six leaf stage. Black necrosis of the plant collar, spreading into leaf midribs, often extending to major veins, accompanied by leaf deformation and growth reduction are observed (Fig. 1). No symptoms are observed on plant roots while the entire above ground vegetation may become necrotic in the more severe cases. The number of affected plants remains low, reaching generally between one and up to 20 plants per square meter, which translates into less than $2 \%$ infected plants given the very high plantation densities used. However, given that harvesting and conditioning are automated, affected plants are difficult to eliminate. The low percentage of infected plants is therefore sufficient to cause the rejection of entire production lots and thus the loss of the entire harvest, severely compromising the profitability and long-term sustainability of corn salad production.

Following initial observations, efforts were developed to understand the origin of these novel symptoms. Given the necrotic symptoms at the collar, initial efforts tried to evaluate the possible contribution of known corn salad pathogens (Rhizoctonia, Thielaviopsis basicola, and Phoma valerianellae) (Stanghellini et al. 1990) and later the possible involvement of other fungi, bacteria, or nematodes. These attempts yielded negative or inconclusive results 
and a possible viral etiology was then investigated, despite the fact that only a single virus had been reported so far in corn salad (Provvidenti 1979). Using a variety of complementary approaches, we report here on the identification of several necroviruses as the causal agents of this emerging disease.

\section{MATERIALS AND METHODS}

Virus isolates and plant samples. A first virus isolate (E1168) was obtained from a naturally infected corn salad plant presenting severe systemic necrotic symptoms and stunting, collected in August 2011 near Nantes, Western France. Infected leaves were triturated with $0.03 \mathrm{M} \mathrm{Na}_{2} \mathrm{HPO}_{4}$ containing $0.2 \%$ Na-diethyldithiocarbamate (DIECA) (1:5, wt/vol) with a mortar and pestle. The homogenate was mixed with 400-mesh Carborundum $(75 \mathrm{mg} / \mathrm{ml})$ and activated charcoal $(75 \mathrm{mg} / \mathrm{ml})$ before being rubinoculated on test plants. Subsequently, a total of 143 additional samples of symptomatic corn salad were obtained from 13 different farms located near Nantes. Tobacco necrosis virus D isolate NZ (TNVD-NZ) originally isolated from lettuce roots (Smith et al. 1969) was provided by R. N. Campbell (University of California, Davis) and tobacco necrosis virus A (TNVA) isolate 87-13 was obtained from a cucumber plant growing near Orléans, Western France (Campbell et al. 1990).

In order to perform host range studies with isolates representing a homogeneous population, an isolate representative of each of the four tentatively identified viruses was first submitted to a series of three successive single local lesion transfers by mechanical inoculation on P. vulgaris or Vigna sinensis. Isolates 2012-H-91 (TNVA), 2012-H-98 (olive mild mosaic virus, OMMV), 2012-K-96 (TNVD), and 2012-I-103 (corn salad necrosis virus, CSNV) were then used to mechanically inoculate an extensive host range of 28 plant species that were monitored for symptom development and tested for virus accumulation.

Serological tests. Crude polyclonal antisera against TNV-KA (a TNVA isolate) and TNV-NZ (a TNVD isolate) were provided by R. N. Campbell (University of California, Davis). Immunoglobulin $\mathrm{G}(\mathrm{IgG})$ and alkaline phosphatase conjugated $\mathrm{IgG}$ were prepared according to standard procedures and used for double antibody sandwich enzyme-linked immunosorbent assay (DAS-ELISA). Samples were ground in the inoculation buffer at 1:10 (wt/vol) and $\mathrm{IgG}$ and alkaline phosphatase conjugated- $\mathrm{IgG}$ (each at $1 \mathrm{mg} / \mathrm{ml}$ ) were used at dilutions 1:250 and 1:1,000 for TNV-KA and TNV-NZ antisera, respectively.

Host range study. The susceptibility of 28 plant species belonging to 10 families was evaluated following mechanical inoculation of two plants for each species. The presence of the virus at the plant apex, 3 weeks after inoculation, was checked by DAS-ELISA using the appropriate antibodies, or by symptoms only when specific antibodies were not available. The host range study was repeated twice.

Electron microscopy. Crude extracts were observed with a Philips CM10 (Eindhoven, The Netherlands) electron microscope using $1 \%$ parlodion and carbon-coated grids and $1 \%$ ammonium molybdate, $\mathrm{pH} 7$, as negative stain.

Complete genome sequences determination by next generation sequencing (NGS). Double-stranded RNAs (dsRNA) were extracted according to the protocol detailed in Candresse et al. (2013) from fresh symptomatic leaves of Nicotiana benthamiana inoculated with sap from symptomatic corn salad. After a whole genome amplification, cDNA libraries prepared from dsRNA samples were submitted to pyrosequencing in a multiplex strategy as described (Candresse et al. 2013). Following demultiplexing and quality trimming, the reads were analyzed using CLC Genomics Workbench 6.5 (https://www. qiagenbioinformatics.com). Assembled reads were annotated by BlastN and BlastX analyses using a cut-off value of $10^{-3}$. Contigs were mapped on TNVA, OMMV, and satellite tobacco necrosis virus (STNV) reference genomes to construct scaffolds for each virus. Determination of the $5^{\prime}$ and $3^{\prime}$ ends of the genomes was performed using specific primers designed from pyrosequencing data or from alignments of viral reference sequences (Supplementary Table S1).

In order to obtain the complete genome sequence of CSNV, small RNAs (sRNAs) were sequenced on an Illumina MiSeq sequencer at Fasteris (Plan-les-Ouates, Switzerland). After total RNA extraction from leaves of $N$. benthamiana inoculated with symptomatic corn salad, small RNAs were purified following polyacrylamide gel electrophoresis and sequenced. The 21 to 24 nucleotide (nt) reads were analyzed as indicated above. Specific primers were designed to determine $5^{\prime}$ and $3^{\prime}$ ends and resequence a central genome portion. The genome extreme $5^{\prime}$ end was further confirmed by rapid amplification of cDNA ends (RACE) (Takara Bio Europe/Clontech, Saint-Germain-en-Laye, France) using a specific reverse primer designed on the sequence of resequenced genomic contig. In order to confirm the genome extreme $3^{\prime}$ end, total nucleic acids extracted from plant 2012-I-103 $(20 \mu \mathrm{g})$ were first polyadenylated (Ambion/ ThermoFisher Scientific, Illkirch, France) and then submitted to reverse transcription using LD-polyT as reverse primer. The 3' region was then amplified using a forward internal primer designed from the resequenced contig and the LD-prime primer, following the protocol of Youssef et al. (2011).

Nucleic acid extraction and detection of the various necroviruses by RT-PCR. Leaves $(30 \mathrm{mg}$ ) were harvested and immediately frozen in liquid nitrogen for RNA isolation. Extraction of RNA was carried out using the Nucleospin RNA plant kit according to the manufacturer instructions (Macherey Nagel, Duren, Germany). Detection of the four viruses as well as the

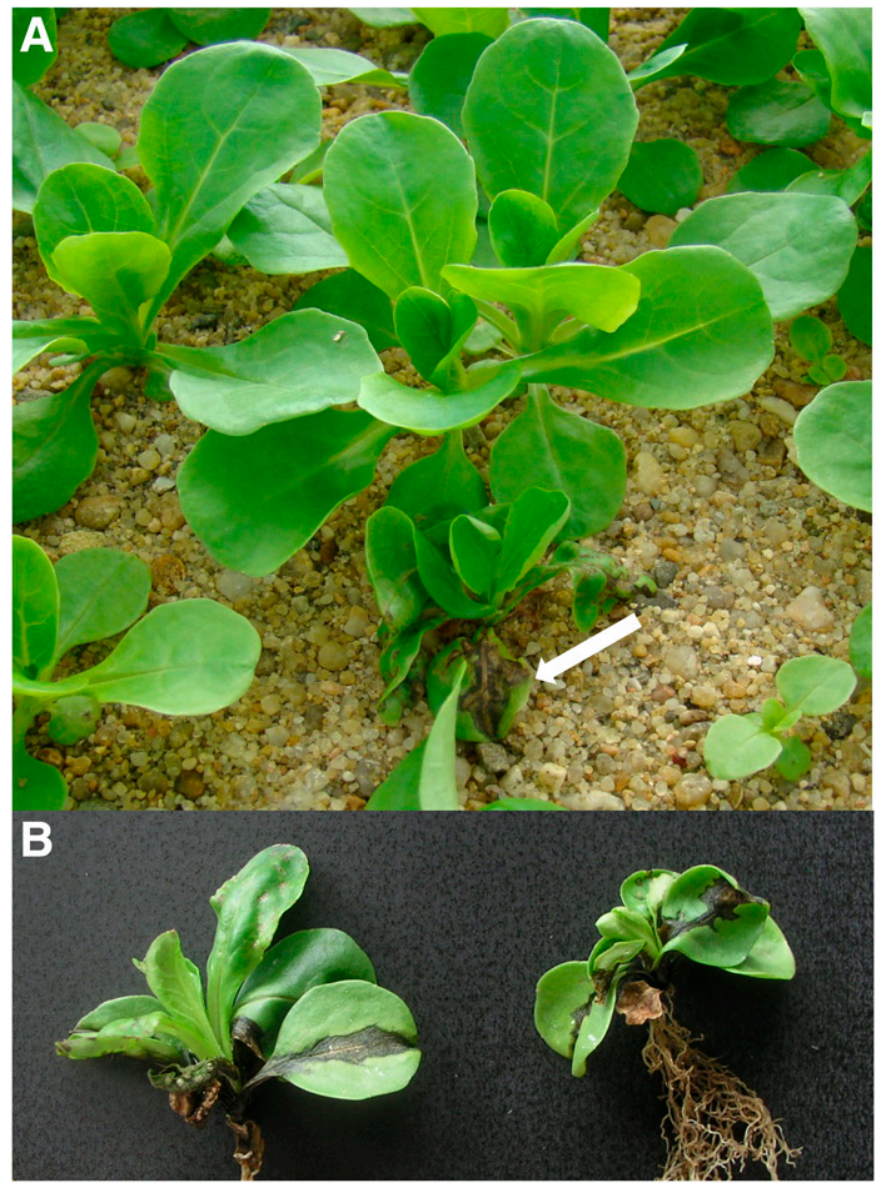

Fig. 1. Symptoms of the systemic necrosis disease on corn salad. A, Symptoms observed in the field. The white arrow points to the leaf of an affected dwarfed plant, showing veinal necrosis of the midrib and of primary veins. B, Symptoms close-up showing systemic midrib necrosis. 
satellite virus was performed using two-step RT-PCR: cDNA was prepared with Superscript II using manufacturer's instructions (ThermoFisher) and $\mathrm{pdN}_{6}$ primers. Sequences of primer used for detection purposes are reported in Table 1. PCR analyses were performed using $10 \mathrm{ng}$ of RNA, $0.25 \mu \mathrm{M}$ of primers, $125 \mu \mathrm{M} \mathrm{dNTP}$, $2.5 \mathrm{mM} \mathrm{MgCl}_{2}$ (for TNVA and TNVD) or $1 \mathrm{mM} \mathrm{MgCl}_{2}$ (for OMMV and STNV), 1 U of Diamond Taq (Life Science), and 1× Taq buffer. PCR cycling was comprised of $15 \mathrm{~min}$ at $95^{\circ} ; 45$ cycles at $94^{\circ}$ for $30 \mathrm{~s}$, using the annealing conditions described in Table $1,72^{\circ}$ for $60 \mathrm{~s}$; and $72^{\circ}$ for $5 \mathrm{~min}$. PCR products were separated on a $1.5 \%$ agarose gel. Uncloned PCR products were sequenced on both strands (GATC Biotech, Köln, Germany).

Sequence comparisons and phylogenetic analyses. Multiple sequence alignments were performed using ClustalW (Thompson et al. 1994) implemented in MEGA version 6 (Tamura et al. 2013). Phylogenetic trees were reconstructed using the neighbor-joining method with strict nucleotide or amino acid distances and randomized bootstrapping for the evaluation of branch validity. Genetic distances ( $p$-distances calculated on nucleotide or amino acid identity) were calculated using MEGA version 6.

\section{RESULTS}

First identification of necroviruses in corn salad. When sap extracts from corn salad sample E11-68 were mechanically inoculated to a limited number of herbaceous hosts, necrotic local lesions, and systemic necrotic symptoms were observed in corn salad and $N$. benthamiana while only necrotic local lesions were observed in bean, zucchini squash and $N$. tabacum 'Xanthi'. The very fast onset of necrotic reaction and the range of symptoms observed were compatible with the involvement of a necrovirus such as tobacco necrosis virus, and a verification of this hypothesis using a serological approach was therefore undertaken. DASELISA tests conducted on the original sample and on mechanically inoculated, symptomatic corn salad and $N$. benthamiana plants gave a positive reaction for TNVD and a negative reaction for TNVA. To further characterize the virus in the sample, primers were designed to detect two genomic regions in the RNA-dependent RNA polymerase (RdRp) and coat protein (CP) genes of TNVA or TNVD (Table 1). RT-PCR products of expected sizes were obtained only with the RdRp primers specific for TNVA and CP primers specific for TNVD, suggesting that OMMV, a natural recombinant between TNVA and TNVD, was likely responsible for the necrotic symptoms observed in corn salad sample E11-68.

In parallel, an independent sample of symptomatic corn salad was analyzed by high-throughput sequencing of cDNAs prepared from purified double-stranded RNAs (dsRNAs). The 454 pyrosequencing reads obtained were cleaned, assembled into contigs and annotated, revealing contigs with high homology with two necroviruses and one satellite virus. Thirteen contigs representing a total of 1,812 reads ( $34.7 \%$ of viral reads) had BLAST hits with OMMV, 1,643 reads (31.5\%) assembled into eight contigs with highest sequence similarity to TNVA, and 13 contigs comprising 3,430 reads $(65.7 \%)$ were annotated as STNV. Because OMMV is a natural recombinant derived from TNVA and therefore shares genomic regions with it (Cardoso et al. 2005), some reads mapped simultaneously on contigs corresponding to both genomes, resulting in a total number of viral reads in excess of $100 \%$. No other virus could be detected among the dsRNA-derived sequencing reads.

A large scaffold of 3,624 nt was reconstructed for the OMMV isolate, for which only $63 \mathrm{nt}$ at the $5^{\prime}$ end were missing. The obtained scaffold for TNVA comprised its genome from nucleotide 605 to nucleotide 3188 , missing 604 nt at the $5^{\prime}$ end and $499 \mathrm{nt}$ at the $3^{\prime}$ end. For STNV, the scaffold obtained comprised 1,202 nt, missing only the first $5^{\prime} 37 \mathrm{nt}$. Considering the very high level of identity $(98.9 \%$ ) (Table 2) between the corn salad STNV isolate and the STNV reference genome (V01468), the missing 5' end of the genome was not determined. Polishing and completion of the genomes of TNVA and OMMV corn salad isolates were performed using the dsRNA extract as template and specific primers designed from pyrosequencing data. The complete sequences of the genomes of the corn salad OMMV, TNVA, and STNV isolates have been deposited in GenBank under accession numbers KX906929, KX906928, and KX906930, respectively.

To confirm the presence of necroviruses in the original pyrosequenced corn salad sample, they were transmitted by mechanical inoculation to $N$. tabacum 'Xanthi', resulting in the expected fast-onset necrotic local lesion symptoms. Leaf dip preparations of a symptomatic tobacco and from the original corn salad sample were examined by electron microscopy, revealing spherical particles of ca. $30 \mathrm{~nm}$ in diameter in both samples (data not shown), a size normally associated with necrovirus particles. Smaller spherical particles of ca. $17 \mathrm{~nm}$ in diameter, a size corresponding to

TABLE 1. Primers used for the detection of different necroviruses and of satellite tobacco necrosis virus (STNV) in the present study

\begin{tabular}{|c|c|c|c|c|c|c|}
\hline Primer name & Sequence $5^{\prime}-3^{\prime}$ & Virus targeted & $\begin{array}{c}\text { Gene } \\
\text { targeted }\end{array}$ & $\begin{array}{l}\text { Genome } \\
\text { positions }\end{array}$ & $\begin{array}{c}\text { Amplicon size } \\
\text { (bp) }\end{array}$ & $\begin{array}{l}\text { Annealing } \\
\text { temperature }\end{array}$ \\
\hline TNVcons-f & GGGGMYTRRTGGAAMGAKTAT & OMMV, TNVA, TNVD, CSNV & $\operatorname{RdRp}$ & $845-865$ & 698 & $57^{\circ} \mathrm{C}$ \\
\hline TNVcons-r & CATGTCHCCRSWCATYCTRCAWCCC & & & $1542-1518$ & & \\
\hline GPINT5 $^{\mathrm{b}}$ & GTGTTCAGTCATATACATACC & OMMV, TNVD & $\mathrm{CP}$ & $3024-3044$ & 256 & $57^{\circ} \mathrm{C}$ \\
\hline GPINT3 $^{b}$ & GCCTATTGTGCTGTACCAC & & & $3279-3262$ & & \\
\hline Sat-14F & TCATTCCGAGTCTCTTGGTCAT & STNV & $3^{\prime} \mathrm{NCR}$ & 890-911 & 146 & $60^{\circ} \mathrm{C}$ \\
\hline Sat-14R & TGGTCCAAACAACGATATGCCG & & & $1035-1014$ & & \\
\hline Sat-14NF & ATTAGTAGGTCTAGCACTCAAAG & STNV & $3^{\prime} \mathrm{NCR}$ & 918-940 & 96 & $50^{\circ} \mathrm{C}$ \\
\hline Sat-14NR & GCGCACTTAATATACCGCTT & & & $1013-994$ & & \\
\hline TNVA-F & CGGATTGTCCGGTAGAGGG & TNVA & $\mathrm{CP}$ & $2778-2796$ & 193 & $50^{\circ} \mathrm{C}$ \\
\hline TNVA-R & TGGTGCTGCTGCAATCAGTGC & & & $2970-2950$ & & \\
\hline TNVA-NF & CTACATAGTGGCTCCCACCT & TNVA & $\mathrm{CP}$ & 2799-2818 & 134 & $52^{\circ} \mathrm{C}$ \\
\hline TNVA-NR & ATGCGCCTAGTGCCGCTAAG & & & $2932-2913$ & & \\
\hline TNVD-polF & CGGCGGGGACTAGTGGAACG & TNVD & $\operatorname{RdRp}$ & 819-838 & 703 & $52^{\circ} \mathrm{C}$ \\
\hline TNVD-polR & CACCAGACATCCTGCATCCC & & & $1521-1502$ & & \\
\hline TNVA-CPf & CGGATTGTCCGGTAGAGGGGG & TNVA & $\mathrm{CP}$ & $2777-2797$ & 444 & $57^{\circ} \mathrm{C}$ \\
\hline TNVA-CPr & ATGGCTGACCCAGCTCCCTGG & & & $3220-3200$ & & \\
\hline $\begin{array}{l}\text { TNVAOMMV- } \\
\text { polF }\end{array}$ & CCAAGGGGAGGGGTGGTGG & OMMV, TNVA, CSNV & RdRp & $596-614$ & 674 & $57^{\circ} \mathrm{C}$ \\
\hline $\begin{array}{l}\text { TNVAOMMV- } \\
\text { polR }\end{array}$ & CTTTGAAGACCGTGATTTCCCC & & & $1269-1248$ & & \\
\hline
\end{tabular}

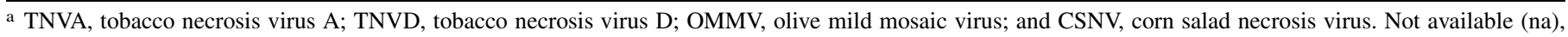
noncoding region (NCR), RNA-dependent RNA polymerase (RdRp), open reading frame (ORF), and coat protein (CP).

b Cardoso et al. (2004). 
that of STNV (Kassanis 1970b), were observed only in the original corn salad sample analyzed by NGS (data not shown).

Taken together these results suggested that several necroviruses, including at least OMMV and TNVA, sometimes in association with STNV, could be responsible for the symptoms observed in corn salad.

Serological characterization of the viruses causing systemic necrosis in corn salad. When the 143 additional symptomatic corn salad samples were tested by DAS-ELISA, 99 reacted positively for TNVD, 51 for TNVA, 17 for both TNVD and TNVA, and 10 gave negative reactions with both antisera. Of the 10 samples testing negative in DAS-ELISA, five were mechanically inoculated to a limited host range and caused similar reactions to those initially observed with OMMV. To further characterize the viral isolates involved, a subsample of 54 isolates giving single reactions in ELISA was submitted to RT-PCR using the four primers sets defined to detect the RdRp and CP coding sequences of TNVA and TNVD. They were all tested in parallel using a necrovirus generic primer pair (Tables 1 and 2). Discounting situations of mixed infection, four different viral entities could be identified in this way (Table 3): OMMV (typified by sample 2012-H-98), TNVA (typified by 2012H-91), TNVD (typified by 2012-K-96), and a fourth virus tentatively named corn salad necrosis virus (CSNV) typified by sample 2012-I-103. CSNV was unusual in (i) not reacting with antibodies against TNVA or TNVD and (ii) being amplified by the primer pair specific for TNVA RdRp but not amplified by the two primer pairs specific for the CP of TNVA or TNVD (Table 3). This novel behavior suggested unusual properties or molecular characteristics of the CSNV CP gene and encoded protein.

Host range of the four viruses and completion of the Koch postulates. An isolate representative of each of the four tentatively identified viruses was submitted to a series of three successive single local lesion transfers on P. vulgaris or Vigna sinensis, and used for further host range and molecular studies. Isolates 2012-H-91 (TNVA), 2012-H-98 (OMMV), 2012-K-96 (TNVD), and 2012-I-103 (CSNV) were inoculated to an extensive host range of 28 different plant species belonging to 10 different genera (Supplementary Table S2). Three weeks after inoculation, necrotic local lesions and systemic necrotic symptoms were observed on all corn salad-inoculated plants (Fig. 2) and the four isolates were detected by DAS-ELISA or RT-PCR and reisolated on test plants (data not shown). This fulfilled Koch's postulates and confirmed that all four viruses were able to cause the necrotic symptoms initially observed in $V$. locusta and that had prompted this investigation. Necrotic local lesion and systemic necrotic symptoms were also observed in all inoculated $N$. benthamiana plants. In $P$. vulgaris, necrotic local lesions were observed on all inoculated plants, but occasionally a few plants developed necrotic streaks on the main stem. Twenty-one plant species developed only necrotic local lesions and no systemic symptoms with the four isolates. Isolates 2012-H-91 2012-H-98 and 2012-K-96 were not detected by DAS-ELISA in the plant apex. Three species (lettuce, tomato, and turnip) developed necrotic local lesions and no systemic infection with only one or two isolates while two species (Physalis floridana and Capsella bursa-pastoris) did not develop symptoms of local or systemic infection with the four isolates.

Interestingly, when TNVD isolate NZ (isolated from lettuce roots) and TNVA isolate 87-13 (isolated from cucumber leaves) were inoculated to corn salad, they induced only necrotic local lesions and no systemic infection (no symptoms and negative reaction in DAS-ELISA), except in rare cases where very few necrotic lesions were observed on noninoculated leaves (not shown). This indicates that the necrovirus isolates obtained from corn salad differ from the known TNVD and TNVA isolates in their ability to systemically invade this novel host.

To compare the severity of the systemic symptoms caused by the four necroviruses on corn salad, six commercial cultivars (Agathe, Baron, Cirilla, Gala, Grosse naine, and Juvalon) were inoculated and symptoms were rated from 0 (no systemic symptoms) to 5 (severe systemic necrotic symptoms) 3 weeks after inoculation. Systemic infection was observed in all cultivars and no significant difference was observed between cultivars (data not shown). In contrast, isolates formed different groups according to NewmanKeuls test (De Muth 2006). Mean ratings on the six cultivars were 3.9 for 2012-H-98 (OMMV), 2.9 for 2012-H-91 (TNVA), 2.4 for 2012-I-103 (CSNV), and only 1.8 for 2012-K-96 (TNVD).

Analysis of the genomic sequences of TNVA and OMMV corn salad isolates. The genomes of the OMMV (3,687 nt) and TNVA $(3,686 \mathrm{nt})$ isolates characterized in the present work have sizes similar to those of the corresponding reference isolates

TABLE 2. Percentages of identity in nucleotides and amino acids (in bold) between the corn salad isolates of olive mild mosaic virus (OMMV), tobacco necrosis virus A (TNVA), and satellite tobacco necrosis virus (STNV) and, for each agent, the corresponding reference sequence ${ }^{\mathrm{a}}$

\begin{tabular}{lccccccc}
\hline Reference isolate & Complete genome & $5^{\prime} \mathrm{NCR}$ & ORF1 (RdRp) & ORF2 (MP1) & ORF3 (MP2) & ORF4 (CP) & $3^{\prime}$ NCR \\
\hline OMMV AY616760 & $90.1 \%$ na & $96.6 \%$ na & $90.6 \% \mathbf{9 6 . 5 \%}$ & $95.9 \% \mathbf{9 7 . 3 \%}$ & $92.9 \% \mathbf{1 0 0 \%}$ & $85.7 \% \mathbf{9 6 . 6 \%}$ & $93.7 \%$ na \\
TNVA M33002 & $98.0 \%$ na & 100.0 na & $98.3 \% \mathbf{9 9 . 0 \%}$ & $99.5 \% \mathbf{1 0 0 \%}$ & $98.2 \% \mathbf{1 0 0 \%}$ & $96.3 \% \mathbf{9 8 . 2 \%}$ & $95.1 \%$ na \\
STNV V01468 & $98.9 \%$ na & na & na & na & na & $99.0 \%$ 99.0\% & $98.9 \%$ na \\
\hline
\end{tabular}

a Not available (na), noncoding region (NCR), RNA-dependent RNA polymerase (RdRp), open reading frame (ORF), movement proteins 1 and 2 (MP1 and MP2), and coat protein $(\mathrm{CP})$.

b Thirty-seven nucleotides are missing at the $5^{\prime}$ end of the STNV genome. The comparisons were performed with the available sequences.

TABLE 3. Detection of the four necroviruses from corn salad using double antibody sandwich enzyme-linked immunosorbent assay (DAS-ELISA) and reverse transcription (RT)-PCR tests

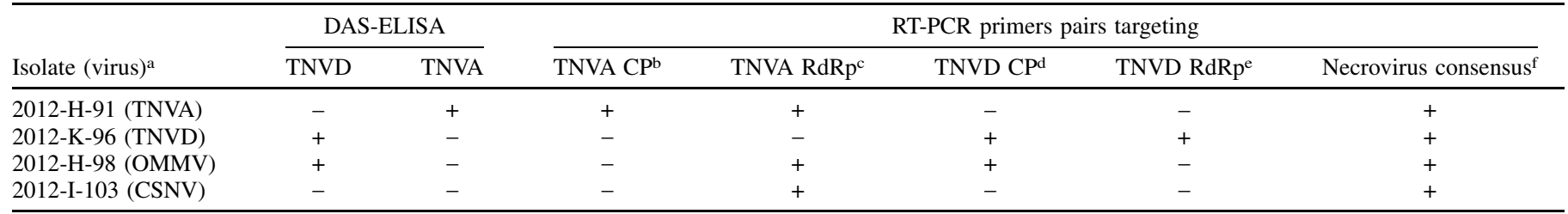

a Tobacco necrosis virus A (TNVA), tobacco necrosis virus D (TNVD), olive mild mosaic virus (OMMV), and corn salad necrosis virus (CSNV). Primer sequences described in Table 1.

b TNVA-CPf/TNVA-CPr.

c TNVAOMMV-polF/TNVAOMMV-pol-R.

d GP1INT5/GP1INT3.

e TNVD-polF/TNVD-polR.

f TNVcons-f/TNVcons-r. 


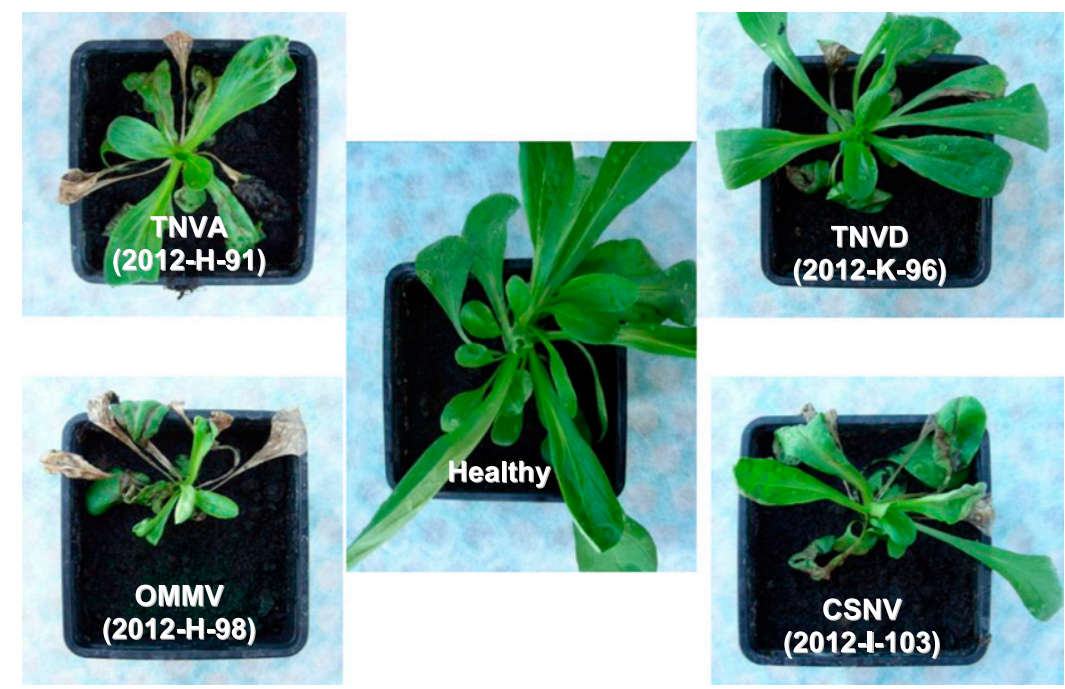

Fig. 2. Necrosis on corn salad 3 weeks after the mechanical inoculation of the corn salad isolates of tobacco necrosis virus A (TNVA) (2012-H-91), tobacco necrosis virus D (TNVD) (2012-K-96), olive mild mosaic virus (OMMV) (2012-H-98), and corn salad necrosis virus (CSNV) (2012-I-103).

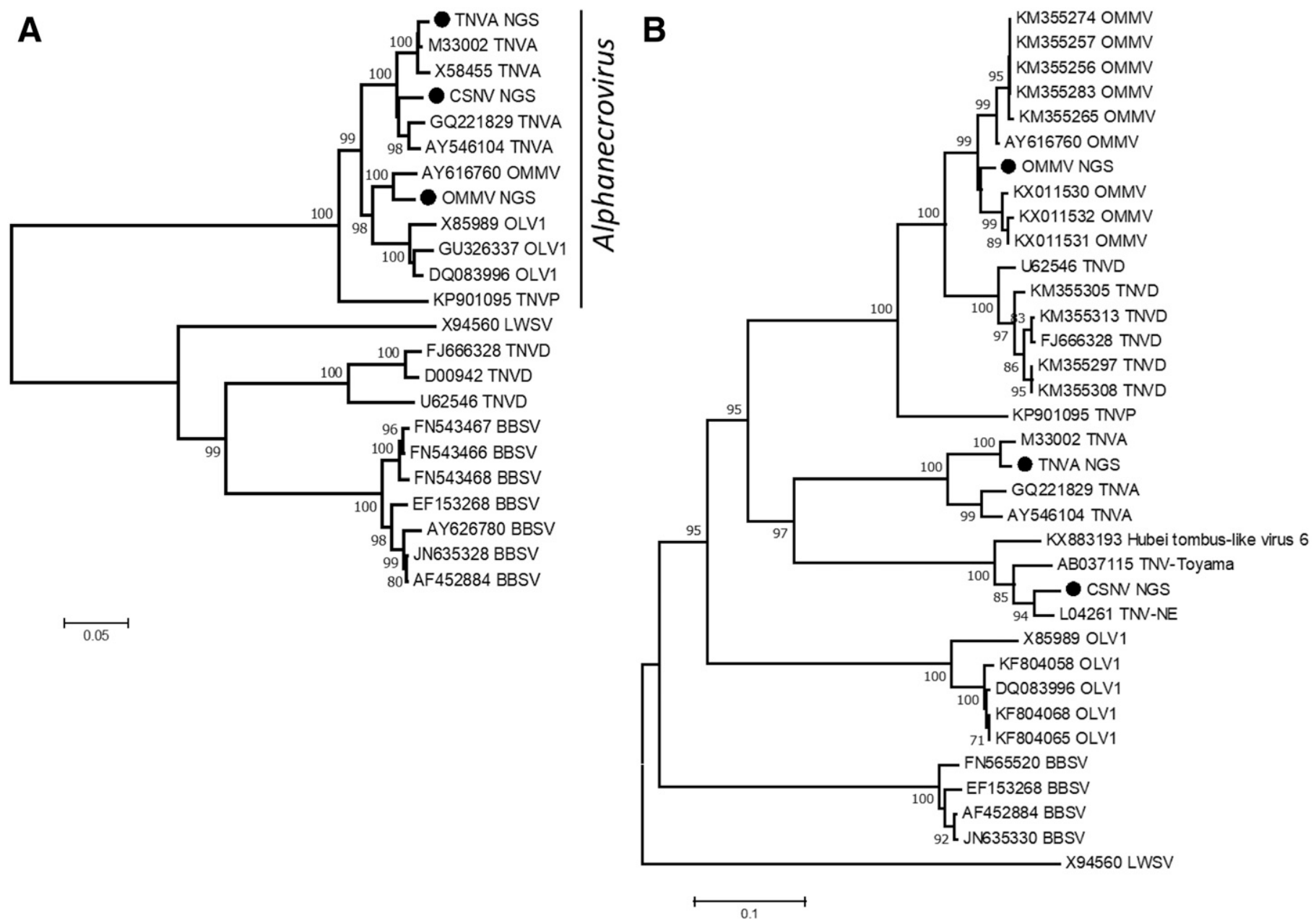

Fig. 3. Phylogenetic analysis of the A, complete polymerase and $\mathbf{B}$, coat protein amino acid sequences from representative species in the family Tombusviridae. The trees were constructed using the neighbor-joining method and the statistical significance of branches was evaluated by bootstrap analysis (1,000 replicates). Only bootstrap values above 70\% are indicated. The scale bar represents 5\% (A) or 10\% amino acid divergence (B) and the sequences of the viruses determined in the present work are marked by a black dot. The abbreviations following the accession numbers are TNVA, tobacco necrosis virus A; TNVD, tobacco necrosis virus D; OMMV, olive mild mosaic virus; TNVP, tobacco necrosis virus P; BBSV, beet black scorch virus; LWSV, leek white stripe virus; OLV1, olive latent virus 1 ; and CSNV, corn salad necrosis virus. 
(AY616760, 3,683 nt and M33002, 3,684 nt, respectively). They have an organization showing the four open reading frames (RdRp, movement proteins [MP] 1 and 2, and CP) typical for members of the newly created genus Alphanecrovirus in the family Tombusviridae (Rochon et al. 2011). In the case of OMMV, the corn salad isolate is highly colinear with the reference isolate; the open reading frames (ORFs) sizes are strictly conserved and only four indel mutations are observed, in the $3^{\prime}$ noncoding region (NCR, $2 \mathrm{nt}$ longer) and in the short intergenic regions between ORF2 and ORF3, and ORF3 and ORF4, respectively (one nt each). The overall nucleotide identity between the corn salad isolate and OMMV reference isolate is $90.1 \%$ (Table 2) and at the amino acid level, the identity between the different viral proteins varies from 96.5 to $100 \%$ (Table 2). In the case of the corn salad TNVA isolate, sequence conservation with the reference isolate is even higher, with amino acid identity values ranging from $98.2 \%$ (ORF4, CP) to $100 \%$ (ORFs 3 and 4, MP1 and MP2) (Table 2). However, the short hypothetical ORF5, which encodes a 59-amino acid (aa) long protein in the reference isolate is truncated in the corn salad isolate because of a $\mathrm{G}$ insertion at position 3507 which creates a premature stop codon, leading to an encoded protein of only 19 aa. Finally, the genome of the STNV corn salad isolate is fully colinear with that of the reference isolate (V01468) and encodes a single protein (putative CP of $196 \mathrm{aa}$ ) which shares $99 \%$ of aa identity with the corresponding protein of reference isolate (Table 2). Phylogenetic analyses performed with the complete polymerase or the $\mathrm{CP}$ amino acid sequences of various necroviruses confirmed the taxonomical position of the corn salad OMMV and TNVA isolates (Fig. 3A and B).

Analysis of the genomic sequence of CSNV. The genome of CSNV was determined from high-throughput siRNAs sequencing data and polished by targeted PCR (described in Materials and Methods). Genome ends were confirmed by RACE ( $5^{\prime}$ end) and a specific PCR on $3^{\prime}$ polyadenylated genomic RNA (described in Materials and Methods). The completed sequence has been deposited as GenBank accession number MF125267. It also shows the typical genomic organization of alphanecroviruses, with four ORFs that are essentially colinear with those of TNVA. The closest relative of CSNV appears to be TNVA with an overall $80 \%$ identity with the M33002 reference isolate (Table 4). Analysis of individual ORFs shows a more complex and contrasted picture. ORF1 (RdRp), 2 and 3 (putative movement proteins) show a high level of identity with TNVA, with 96.1, 91.8, and $98.2 \%$ aa identity, respectively, of the encoded proteins (Table 4). Indeed, a phylogenetic analysis shows the CSNV RdRp clusters with high bootstrap support with the corresponding protein from TNVA isolates (Fig. 3A), and this level of divergence falls within the $93 \%$ identity cut-off value currently accepted for species discrimination in the family (Rochon et al. 2011). The CP is however highly divergent with that of TNVA, showing only about $53.6 \%$ nt identity (53\% aa identity) (Table 4), a value which is under the $55 \%$ aa identity species discrimination criterion (Rochon et al. 2011). The phylogenetic analysis further supports, with high bootstrap validation, that corn salad necrosis virus is a new species of alphanecrovirus (Fig. 3B).

On the other hand, CSNV shows a very high level of identity with the partial sequence of a TNV isolate from Nebraska (L04261) (Zhang et al. 1993) with amino acid identity levels of between 95.9 and $100 \%$ (95.9 to $98.8 \%$ nt identity) for the proteins encoded by
ORFs 2, 3, and 4 (Table 4). CSNV CP is also highly similar $(90.3 \%$ aa identity) to the CP of TNV-Toyoma (AB037115) (Saeki et al. 2001) (Fig. 3B).

Presence of possible recombination events in a dataset composed of aligned complete TNVA, TNVD, OMMV, and CSNV genomic sequences was evaluated using the various programs implemented in RDP4 (Martin et al. 2005). One recombination event was observed between CSNV and the TNVA/OMMV group, with a recombination breakpoint located between MP2 and CP $\left(P=10^{-27}\right.$ to $P=10^{-75}$ depending on the program used), suggesting that similar to OMMV, CSNV might be a recombinant.

PCR detection and analysis of genetic diversity of necrovirus isolates associated with corn salad. Over a period of 3 years (2011 to 2013), a total of 51 symptomatic plants originating from nine fields were analyzed for the presence of TNVA, OMMV, TNVD, CSNV, and STNV by RT-PCR. OMMV was the most frequently observed virus. It was detected each year and in most of the sampled fields, either in single infection (39/51 infected plants) or in mixed infection (11 cases with TNVA, 1 case with TNVD). Analysis of the 217-nt-long sequences (excluding primer sequences) obtained for 34 isolates using the of the GPINT5/GPINT3 primer pair (corresponding to 137 to 208 aa of the $\mathrm{CP}$, accession numbers KX944577 to KX944610) showed that with one exception, all isolates fell into a single phylogenetic cluster, which also contains a tulip isolate from the Netherlands (EF201605). Two subclusters can be distinguished, one, supported by a $98 \%$ bootstrap value, comprises all but one of the isolates detected in 2011, while the second comprises the isolates collected in 2012 and 2013 (Fig. 4). The genetic diversity of corn salad OMMV isolates is moderate with a maximal $7.4 \%$ nt divergence in the amplified fragment (mean divergence $3.5 \pm 0.8 \%$ ).

TNVA was also frequently recovered, either alone ( 8 cases) or in coinfection with OMMV (11 cases). A phylogenetic analysis performed for 16 isolates, using the 153-nt-long sequences of the TNVA-F/TNVA-R PCR product (excluding primer sequences) which encodes the central part of the $\mathrm{CP}$ (accession numbers KX944613 to KX944628), showed that all isolates clustered in the same tight group together with the original TNVA isolate FM1B (GenBank M33002), collected on tobacco in the UK in the late 1950s (Babos and Kassanis 1963) (Fig. 5). Maximal sequence divergence between corn salad TNVA isolates reached only $0.7 \%$.

The diversity detected for STNV was similarly very limited, with all corn salad isolates forming a very tight cluster (average nucleotide divergence $1.1 \pm 0.6 \%$ ). The limited number of isolates analyzed for TNVD (3) and CSNV (2) precludes any detailed analysis of the variability of these agents in corn salad crops.

\section{DISCUSSION}

The emerging disease of corn salad analyzed here is unusual in several aspects. On corn salad, viral infection had been previously documented in a single case (by Alfalfa mosaic virus) (Provvidenti 1979). So far, the new disease has been observed affecting only a low number of plants in contaminated plots (on the order of 1 to $2 \%$ maximum) but such a low prevalence of infected plants can still result in complete or near complete production loss. Lastly, the results

TABLE 4. Percentages of identity in nucleotides and amino acids (in bold) between the corn salad necrosis virus (CSNV) isolate, and the reference isolates of olive mild mosaic virus (OMMV), tobacco necrosis virus A (TNVA), and tobacco necrosis virus NE (TNV-NE) a

\begin{tabular}{|c|c|c|c|c|c|c|c|}
\hline Reference isolate & Complete genome & $5^{\prime} \mathrm{NCR}$ & ORF1 (polymerase) & ORF2 (MP1) & ORF3 (MP2) & ORF4 (CP) & $3^{\prime} \mathrm{NCR}$ \\
\hline TNVA M33002 & $80.0 \%$ & $90.3 \%$ & $88.9 \% 96$ & $91.0 \% \mathbf{9 1 . 8 \%}$ & $90.5 \% \mathbf{9 8 . 2 \%}$ & $53.6 \% \mathbf{5 3 . 0 \%}$ & $70.6 \%$ na \\
\hline TNV-NE L04261 & $\mathrm{na}^{\mathrm{b}}$ & $\mathrm{na}^{\mathrm{b}}$ & $\mathrm{na}^{\mathrm{b}}$ & $97.0 \% \mathbf{9 5 . 9 \%}$ & $98.8 \% \mathbf{1 0 0 \%}$ & $95.9 \% \quad 96.0 \%$ & $87.4 \%^{\mathrm{c}}$ na \\
\hline OMMV AY616760 & $75.4 \%$ na & $73.4 \%$ na & $83.1 \% \mathbf{9 0 . 6 \%}$ & $85.4 \%$ 73.5\% & $93.5 \% 98.2 \%$ & $49.1 \% \mathbf{4 3 . 4 \%}$ & $73.8 \%$ na \\
\hline
\end{tabular}

a Not available (na), noncoding region (NCR), open reading frame (ORF), movement proteins 1 and 2 (MP1 and MP2), and coat protein (CP).

b Sequence for comparison not available in L04261.

c Partial sequence available in L04261 for comparison only. 
presented here demonstrate that the disease appears to be a syndrome caused by several related necroviruses that share a similar biology.

Four viruses have been identified in symptomatic corn salad plants, in order of decreasing prevalence, OMMV, TNVA, TNVD,

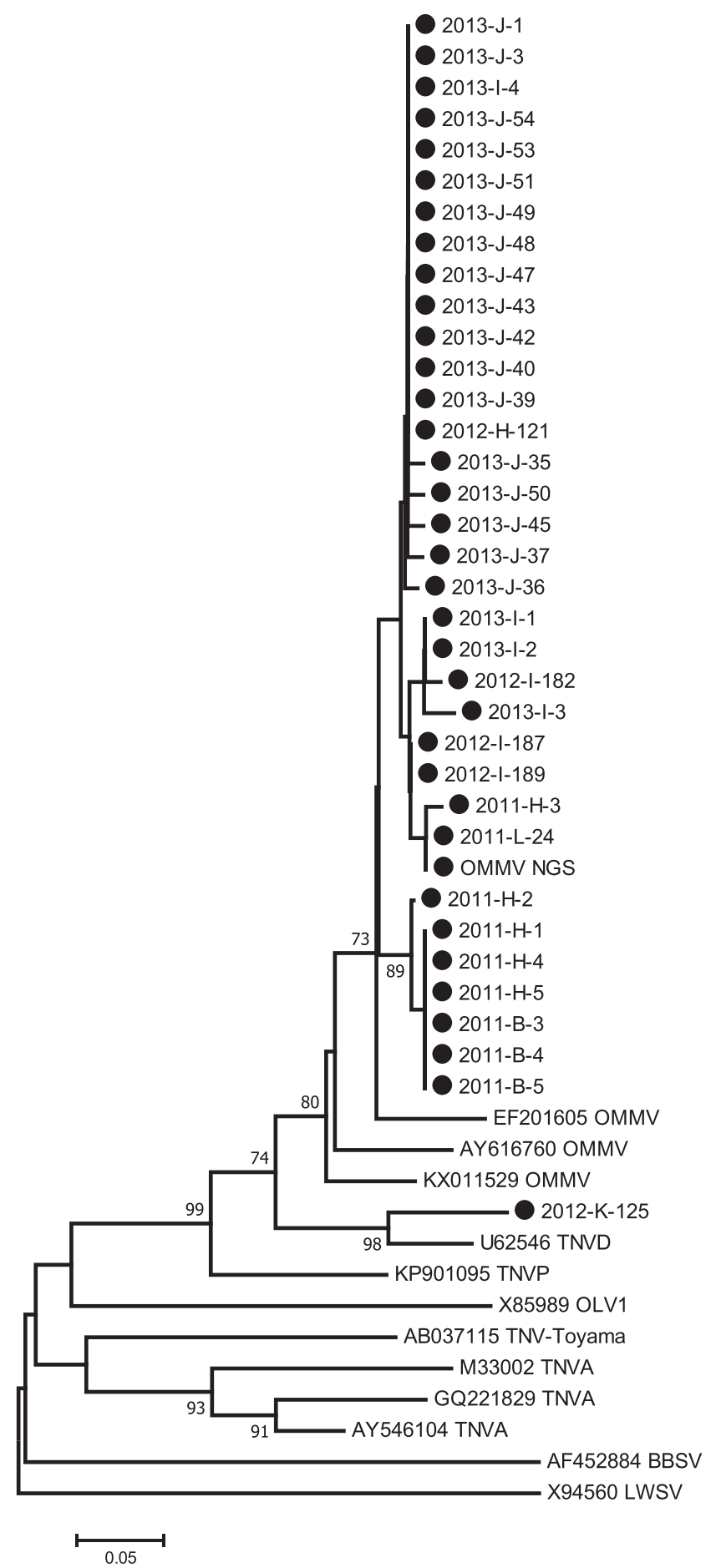

Fig. 4. Unrooted phylogenetic tree reconstructed using the nucleotide sequences from the amplified fragment of the coat protein gene (217 nt, excluding primer sequences) of corn salad isolates of olive mild mosaic virus. The tree was constructed using the neighbor-joining method and the statistical significance of branches was evaluated by bootstrap analysis $(1,000$ replicates). Only bootstrap values above $70 \%$ are indicated. The scale bar represents 5\% nucleotide divergence. The isolates sequenced during the present work are marked by black dots and a detailed listing of these isolates, together with GenBank accession numbers, is provided in Supplementary Table S3. The abbreviations following the accession numbers are TNVA, tobacco necrosis virus A; TNVD, tobacco necrosis virus D; OMMV, olive mild mosaic virus; TNVP, tobacco necrosis virus P; BBSV, beet black scorch virus; LWSV, leek white stripe virus; and OLV1, olive latent virus 1. and CSNV. Tobacco necrosis disease and a virus associated with it were initially described in the 1930s and early 1940s (Bawden 1941; Smith and Bald 1936). The existence of a large variability in the causal agent, in terms of serology, of host specificity and of the ability to support a satellite virus was rapidly reported, resulting in the description of multiple strains (Babos and Kassanis 1963; Kassanis 1970a). Among those, strains A and D were later recognized to be sufficiently distinct and therefore elevated to the status of distinct viral species (Rochon et al. 2011). More recently, as the consequence of the splitting of the genus Necrovirus, they have been separated in different genera, with TNVA typifying the alphanecroviruses and TNVD the betanecroviruses (Rochon et al. 2011). TNVA and TNVD likely share an ancestral recombination event, since their coat proteins are closely related while their polymerases are only distantly related (compare Fig. 3A and B). The complete genome characterization of OMMV, which was previously regarded as a strain of TNVD (Cardoso et al. 2004), lead to the realization that it had a TNVA-like polymerase and a TNVDlike CPs and likely resulted from a recombination event (Cardoso et al.2005). OMMV was therefore elevated to the status of a distinct alphanecrovirus species. Given the currently accepted molecular species discrimination criteria of $93 \%$ aa identity in the polymerase or 55\% identity in the CP (Rochon et al. 2011), it is likely that yet

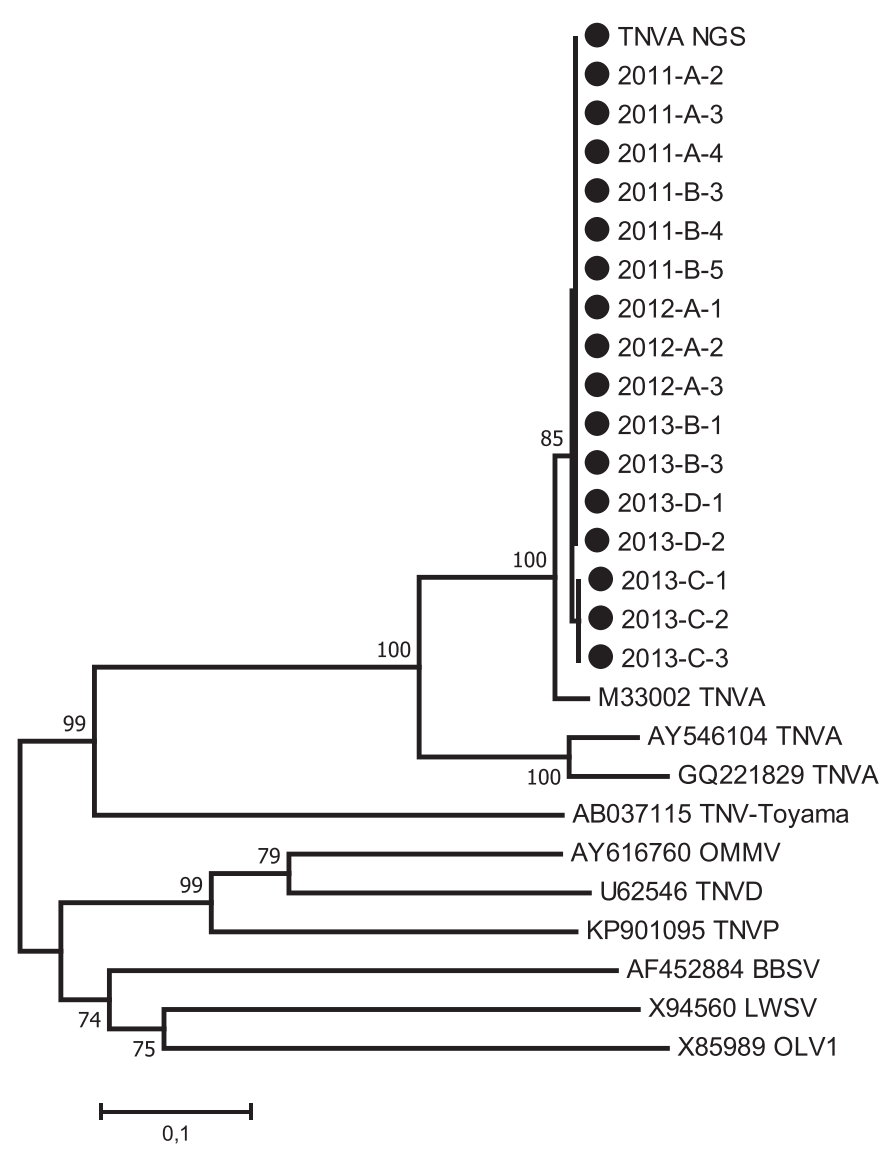

Fig. 5. Unrooted phylogenetic tree reconstructed using the nucleotide sequences from the short amplified fragment of coat protein gene (153 nt, excluding primer sequences) of tobacco necrosis virus $\mathrm{A}$ isolates. The tree was constructed using the neighbor-joining method and the statistical significance of branches was evaluated by bootstrap analysis (1,000 replicates). Only bootstrap values above $70 \%$ are indicated. The scale bar represents $10 \%$ nucleotide divergence. The isolates sequenced during the present work are marked by black dots and a detailed listing of these isolates, together with GenBank accession numbers, is provided in Supplementary Table S3. The abbreviations following the accession numbers are TNVA, tobacco necrosis virus A; TNVD, tobacco necrosis virus D; OMMV, olive mild mosaic virus; TNVP, tobacco necrosis virus P; BBSV, beet black scorch virus; LWSV, leek white stripe virus; and OLV1, olive latent virus 1 . 
other species of necroviruses remain to be described. In particular, the CP sequence of a U.S. isolate from tobacco (L04261, TNV-NE) (Zhang et al. 1993) is highly divergent (ca. 40 to $50 \%$ identity only) from the $\mathrm{CP}$ of all other currently accepted necrovirus species and likely represents such a situation.

The complete genome sequence of CSNV reported here is highly homologous to the 1.6-kb partial sequence of TNV-NE (Table 4; Fig. 3B). However, in the absence of complete sequence information on the TNV-NE polymerase and of the frequent occurrence of recombination in the evolutionary history of necroviruses, it is not possible to conclude that they represent the same agent. Indeed, TNVNE is reported to be serologically related to both TNVA and TNVD in double gel diffusion assays (Zhang et al. 1993), while CSNV failed to react in ELISA with antibodies specific for these viruses. Although this difference may reflect the use of different immunological assays/ reagents, it may also reflect serological differences between the two agents.

Similar to OMMV, CSNV appears to represent a distinct species, likely deriving from a recombination event; while its polymerase is very closely related to that of TNVA $(96.1 \%$ aa identity, within the accepted species variability limit), its $\mathrm{CP}$ is highly divergent from all necroviral CPs, with the exception of that of TNV-NE, and to a lesser extent, of that of a Japanese isolate from tulip (Saeki et al. 2001) (Fig. 3B). We therefore suggest that CSNV should be regarded as a distinct alphanecrovirus species.

It is worth mentioning that another putative viral entity shows a $\mathrm{CP}$ with clear affinities with that of CSNV. It is Hubei tombus-like virus 6 strain WGML12775 (Shi et al. 2016), an agent identified by datamining of transcriptome data from a wide range of invertebrates. The predicted genomic organization of that agent is quite different from that of necroviruses. Its predicted replicationassociated protein is about 45 to $55 \%$ identical to that of the avenavirus oat chlorotic stunt virus, but its predicted CP shows $87.6 \%$ aa identity (73.5\% nt identity) with that of CSNV (Fig. 3B). It is interesting to speculate that such an arthropod-associated virus might either represent the origin of the CSNV CP or, alternatively, that it may have acquired a necroviral CP from a CSNV-related plant virus, in a host interkingdom recombination event.

TNV-related viruses have some important biological characteristics, including their transmission by soil-inhabiting chytridiomycete Olpidium brassicae (Kassanis 1970a; Kassanis and MacFarlane 1964; Rochon et al. 2004; Teakle 1962). Early reports indicated that there might exist some level of specificity in the interaction between TNV strains, fungal isolates, and host species, with a major contribution of the interaction between the zoospores of the fungal vector and the host plant (Kassanis and MacFarlane 1965; Teakle and Hiruki 1964). Recently, two specific amino acids of the CP of OMMV were shown to be involved in transmission by $O$. brassicae (Varanda et al. 2011). Although no specific efforts were made to characterize in detail the Olpidium populations present in the affected corn salad plots, star-shaped resting spores similar to those of O. brassicae sensu lato (Herrera-Vasquez et al. 2009) and zoosporangia were frequently observed in the roots of infected plants (not shown).

Another biological property shared by TNV strains is that they only cause local, nonsystemic infection in most hosts (Kassanis 1970a). The four viruses identified in corn salad fit this picture and indeed infected systemically only a very limited number of the evaluated host species. They all did, however, systemically invade corn salad and cause systemic symptoms in that host, although symptoms severity differed between agents. In contrast, when unrelated TNVA and TNVD isolates were similarly inoculated to corn salad, they produced necrotic local lesions and only very rarely a few systemic necrotic spots. This observation suggests that emergence of the corn salad disease may have necessitated some adaptation of the viral isolates/species involved to this particular host. The short rotation cycle used and the practice of repeated corn salad cropping on individual plots may have contributed to such an adaptation. The identification of the four necroviruses as the causal agents of the disease opens the way to the development and evaluation of control strategies (Campbell et al. 1980; Jones 2004; Smith et al. 1969), including attempts at biological control against the vector, introduction of rotation cycles, management of irrigation, and elimination of viruses by soil desiccation during the intercrop period, some of which appear promising.

\section{ACKNOWLEDGMENTS}

CCDM gratefully thanks the Centre Technique Interprofessionnel des Fruits et Légumes (CTIFL) for hosting CCDM personnel in the Phytopathology laboratory of its Carquefou Research Center. We thank CCDM staff members S. Bosseur, V. Cerceau, M. Lorne, S. Gérard, S. Mével, and P. Verron for contributing to some of the prospections or experiments and Brigitte Batailler and the Plateau d'imagerie du Végétal (Bordeaux) for electron microscope observations; and R. N. Campbell (University of California, Davis) for providing viral isolates and antisera.

\section{LITERATURE CITED}

Babos, P., and Kassanis, B. 1963. Serological relationships and some properties of tobacco necrosis virus strains. J. Gen. Microbiol. 32:135-144.

Bawden, F. C. 1941. The serological reactions of viruses causing tobacco necrosis. Br. J. Exp. Pathol. 22:59-70.

Campbell, R. N., Greathead, A. S., and Westerlund, F. V. 1980. Big-vein of lettuce: Infection and methods of control. Phytopathology 70:741-746.

Campbell, R. N., Lecoq, H., Wipf-Scheibel, C., and Sim, S. T. 1990. Soil-borne viruses of melons and cucumbers in France. Pages 153-154 in: Proc. 1st Symp. Int. Work. Group on Plant Viruses with Fungal Vectors, Braunschweig. Vol. 1. R. Koenig, ed. Ulmer GmbH, Stuttgart, Germany.

Candresse, T., Marais, A., Faure, C., and Gentit, P. 2013. Association of Little cherry virus 1 (LChV1) with the Shirofugen stunt disease and characterization of the genome of a divergent LChV1 isolate. Phytopathology 103:293-298.

Cardoso, J. M. S., Félix, M. R., Clara, M. I. E., and Oliveira, S. 2005. The complete genome sequence of a new necrovirus isolated from Olea europaea L. Arch. Virol. 150:815-823.

Cardoso, J. M. S., Félix, M. R., Oliviera, S., and Clara, M. I. E. 2004. Tobacco necrosis virus D isolate from Olea europaea L.: Viral characterization and coat protein sequence analysis. Arch. Virol. 149:1129-1138.

De Muth, J. E. 2006. Basic Statistics and Pharmaceutical Statistical Applications, 2nd ed. Chapman and Hall/CRC, Boca Raton, FL.

Herrera-Vasquez, J. A., Cebrian, M. C., Alfaro-Fernandez, A., Cordoba-Selles, M. C., and Jorda, C. 2009. Multiplex PCR assay for the simultaneous detection and differentiation of Olpidium bornovanus, O. brassicae and O. virulentus. Mycol. Res. 113:602-610.

Jones, R. A. C. 2004. Using epidemiological information to develop effective integrated virus disease management strategies. Virus Res. 100:5-30.

Kassanis, B. 1970a. Tobacco necrosis virus. AAB Description of Plant Viruses 14. http://www.dpvweb.net/dpv/showdpv.php?dpvno=14

Kassanis, B. 1970b. Satellite virus. AAB Description of Plant Viruses 15. http://www.dpvweb.net/dpv/showdpv.php?dpvno=15

Kassanis, B., and MacFarlane, I. 1964. Transmission of tobacco necrosis virus by zoospores of Olpidium brassicae. J. Gen. Microbiol. 36:79-93.

Kassanis, B., and MacFarlane, I. 1965. Interaction of virus strain, fungus isolate, and host species in the transmission of tobacco necrosis virus. Virology 26:603-612.

Martin, D. P., Williamson, C., and Posada, D. 2005. RDP2: Recombination detection and analysis from sequence alignments. Bioinformatics 21:260-262.

Peron, J. Y., and Rees, D. C. 1998. High-tech production of corn salad (Valerianella locusta (L) Laterr.), a local, French vegetable crop. Acta Hortic.: 259-268.

Provvidenti, R. 1979. Alfalfa mosaic virus infection in Valerianella olitoria. Plant Dis. Rep. 63:820-821.

Rochon, D., Kakani, K., Robbins, M., and Reade, R. 2004. Molecular aspects of plant virus transmission by olpidium and plasmodiophorid vectors. Annu. Rev. Phytopathol. 42:211-241.

Rochon, D., Lommel, S., Martelli, G. P., Rubino, L., and Russo, M. 2011. ICTV taxonomic proposal 2011.009a-mP.A.v3.split_necrovirus. Divide the genus Necrovirus into 2 new genera, Alphanecrovirus and Betanecrovirus. https://talk.ictvonline.org/files/ictv_official_taxonomy_updates_since_the_ 8th_report $/ \mathrm{m} /$ plant-official $/ 4472$

Saeki, K., Takahashi, Y., Oh-Oka, H., Umeoka, T., Oda, Y., and Fukuyama, K. 2001. Primary structure and phylogenetic analysis of the coat protein of a Toyama isolate of tobacco necrosis virus. Biosci. Biotechnol. Biochem. 65: 719-724. 
Shi, M., Lin, X. D., Tian, J. H., Chen, L. J., Chen, X., Li, C. X., Qin, X. C., Li, J., Cao, J. P., Eden, J. S., Buchmann, J., Wang, W., Xu, J., Holmes, E. C., and Zhang, Y. Z. 2016. Redefining the invertebrate RNA virosphere. Nature 540:539-543.

Smith, K. M., and Bald, J. G. 1936. A description of a necrotic virus disease affecting tobacco and other plants. Parasitology 27:231-238.

Smith, P. R., Campbell, R. N., and Fry, P. R. 1969. Root discharge and soil survival of viruses. Phytopathology 59:1678-1687.

Stanghellini, M. E., Rasmussen, S. L., and Barta, D. J. 1990. Thielaviopsis root rot of corn-salad. Plant Dis. 74:81.

Tamura, K., Stecher, G., Peterson, D., Filipski, A., and Kumar, S. 2013. MEGA6: Molecular Evolutionary Genetics Analysis version 6.0. Mol. Biol. Evol. 30:2725-2729.

Teakle, D. S. 1962. Transmission of tobacco necrosis virus by a fungus, Olpidium brassicae. Virology 18:224-231.

Teakle, D. S., and Hiruki, C. 1964. Vector specificity in Olpidium. Virology 24:539-544.
Thompson, J., Higgins, D. G., and Gibson, T. J. 1994. CLUSTALW: Improving the sensitivity of progressive multiple sequence alignment through sequence weighting, position-specific gap, penalties and weight matrix choice. Nucleic Acids Res. 22:4673-4680.

Varanda, C., Felix, M. R., Soares, C. M., Oliveira, S., and Clara, M. I. 2011. Specific amino acids of Olive mild mosaic virus coat protein are involved in transmission by Olpidium brassicae. J. Gen. Virol. 92:2209-2213.

Wikipedia. 2017. Valerianella locusta. https://en.wikipedia.org/wiki/ Valerianella_locusta

Youssef, F., Marais, A., Faure, C., Barone, M., Gentit, P., and Candresse, T. 2011. Characterization of Prunus-infecting Apricot latent virus-like Foveaviruses: Evolutionary and taxonomic implications. Virus Res. 155: 440-445.

Zhang, L., French, R., and Langenberg, W. G. 1993. Molecular cloning and sequencing of the coat protein gene of a Nebraskan isolate of tobacco necrosis virus: The deduced coat protein sequence has only moderate homology with those of strain A and strain D*. Arch. Virol. 132:291-305. 